\title{
Holzgas-Blockheizkraftwerke für Holz und Holzabfälle - Konzepte - Technik - Betriebserfahrung
}

\author{
Dipl.-Phys. Wilfried Richter, Prof. Dr.-Ing. Udo Hellwig
}

\section{Warum nach neuen Energieträgern suchen?}

In der Energiepolitik sind für die Zukunft Ziele gesteckt, die keinen unmittelbaren wirtschaftlichen Gewinn erkennen lassen. Dazu gehört die Reduzierung der $\mathrm{CO}_{2}$ Emissionen, um den Treibhauseffekt zu mindern und die Erschliessung neuer Energiequellen, die notwendig sind, um langfristig die Energieversorgung zu sichern. Die Vorräte an fossilen Energieträgern sind begrenzt. Auf der Suche nach anderen Energieträgern bieten sich Reststoffe aus der Produktion an. Diese Reststoffe verfügen oft über einen ausreichenden Heizwert, so daß sich die energetische Nutzung lohnt. Auf der anderen Seite stellen sie ein Entsorgungsproblem dar, weil die Deponiekapazitäten nicht ausreichen bzw. ihre Deponierung nach dem Gesetz nicht mehr erlaubt ist. Für neue Energieträger müssen neue Energiewandler entwickelt werden. Dabei sind Maschinen, die die hochwertige Energie Strom erzeugen, besonders interessant. Die erforderliche Technik für die Aufgaben der Zukunft muß rechtzeitig entwickelt sein, damit sie zur Verfügung steht, wenn sie gebraucht wird. Der für diese Entwicklung nötige Freiraum ist nur sehr begrenzt. Die Kriterien für die Förderung neuer Techniken sind nicht nach den langfristigen Notwendigkeiten ausgerichtet, sondern orientieren sich an dem kurzfristigen Erfolg.

Hier sehen wir einen Interessenskonflikt, der kaum aufzuheben ist. Politisch wird in einem Vierjahreszyklus geplant, da für die Entscheidungsträger ein Erfolg ihrer Arbeiten danach nicht mehr zumutbar ist. In der Wirtschaft sind die Zyklen durch die Amortisationszeiten bestimmt. Langfristige Perspektiven zeichnen sich nur dort ab, wo der Profit absehbar ist. Ein Beispiel dafuir ist der Ausbau des Erdgasnetzes und dessen Nutzung für die nächsten 50-70 Jahre.

Fast $90 \%$ aller Blockheizkraftwerke werden zur Zeit mit Erdgas oder Heizöl befeuert. Feste Biomasse kommt kaum zum Einsatz. Das Marktsegment für BHKW's mit festen Brennstoffen erscheint den Motorenherstellern zu klein, um in die Entwicklung zu investieren. Dabei steht mit der heimischen Ressource Holz eine große Energiequelle zur Verfuigung.

Allein in Brandenburg ist jährlich eine Holzmenge mit einem Energiepotential von $3500 \mathrm{GWh}$ verfügbar. Der Anteil an Strom, der mit Holzgaserzeugern aus dem Holz gewonnen werden könnte, ist bestimmt durch den elektrischen Wirkungsgrad der Gesamtanlage. Mit dem heute schon realistischem Wirkungsgrad von $25 \%$ kann eine Stromausbeute von $875 \mathrm{GWh}$ erreicht werden. Um dieses Potential auszunutzen, sind allein für Brandenburg Holzgas-Blockheizkraftwerk-Systeme mit einer Leistung von $150 \mathrm{MW}$ notwendig. Das sind nur für Bran- denburg mehr als $15 \%$ der im gesamten Bundesgebiet 1994 installierten BHKW-Kapazitäten mit einer Gesamtleistung von $900 \mathrm{MW}$. Der Markt ist vorhanden, und das bestätigen auch die Anfragen in unserem Haus.

\section{Bezug der heutigen Technik zur Holz- gasära in den 40er Jahren}

Im II. Weltkrieg wurden Holzgaserzeuger eingesetzt, weil fossile Energieträger nicht verfügbar waren. Mit dem Ende des Krieges brach der Absatz von Gaserzeugern zusammen. Die Nachteile waren offensichtlilch. Die Anlagen erforderten einen hohen Service und Wartungsaufwand bei geringer Leistung. So mußten z. B. alle $20.000 \mathrm{~km}$ die Motoren der LKW's überholt werden. Das entspricht einem Wartungsintervall von $600 \mathrm{~h}$ bei heutigen BHKW's. Die 50 Jahre alten technischen Lösungen sind heute nicht mehr akzeptabel. Um ein Holzgaserzeuger-BHKW-System wirtschaftlich sinnvoll betreiben zu können, sind deutlich längere Standzeiten und Wartungsintervalle erforderlich.

Die Kontinuität der Entwicklung von Gaserzeugern ist abgerissen, da die billigen fossilen Energieträger diese Technik vollständig verdrängt haben. Die moderne Technik bietet aber Möglichkeiten in der Filtertechnik und beim Motorenmanagement, die den Holzgaserzeuger wieder konkurrenzfähig machen. Die heutige Entwicklungsarbeit besteht darin, bewährte Gaserzeuger, die teerfreies Gas produzieren, mit den Verfahren der Brennstoffzufuihrung, der Filter- und Motorentechnik zu kombinieren.

\section{Stand der Technik}

Die kostengünstige und umweltfreundliche Produktion von Energie wird zunehmend acu für kleine und mittlere Betriebe gegenüber dem herkömmlichen Energiebezug eine zu prüfende Alternative. Bei der Holzgewinnung und -verarbeitung anfallende Reststoffe sind günstige Energieträger. Die Produktion von Wärme ist mit Holz recht einfach. Komplizierter ist die Stromerzeugung. Gerade im unteren Leistungsbereich bis $500 \mathrm{~kW}_{\mathrm{el}}$ sind bisherige Anlagen (Dampf- und Gasturbinen) wenig effektiv. Bei der Suche nach Alternativen im Energiemanagement bietet sich der Holzgaserzeuger für die dezentrale Energieerzeugung an. Entscheidend für die kommerzielle Nutzung einer Anlage ist aber deren $\mathrm{Zu}$ verlässigkeit und Sicherheit. Daher wurde im TSZ (Trainings- und SchulungsZentrum Fürstenwalde) im Januar 1995 ein Projekt gestartet, in dem ein Holzgaserzeuger im Dauerbetrieb unter den heutigen Bedingungen geste- 
stet wird. Im ersten Betriebsjahr konnten Erfahrungen gesammelt, Daten und Bilanzen erfaßt und ausgewertet werden. Die wesentlichen Erwartungen wurden erfüllt, so daß nach einer weiteren Optimierung eine kommerziell lohnende Alternative in der Energieproduktion besteht.

Anlagen für die kommerzielle Nutzung mit den nötigen Gewährleistungen sind auf dem Markt nicht verfügbar. Es gibt bewährte Anlagen, die in strukturschwachen Ländern eingesetzt werden. Dort spielen Personalkosten und Umweltverträglichkeit, anders als hier, eine unwesentliche Rolle. Daher fehlen Automatisation und Steuerungstechnik. Durch ihre solide Technik bieten solche Holzgaserzeuger aber eine gute Ausgangsbasis für die Weiterentwicklung nach den Erfordernissen am europäischen Markt.

Um diesen Entwicklungsprozeß in Gang zu bringen, wurde am TSZ (Trainings- und SchulungsZentrum Fürstenwalde) ein Projekt begonnen, bei dem ein kleiner Gaserzeuger zunächst ohne Automatisation für die Strom- und Wärmeproduktion eingesetzt wurde. Der Betrieb wird genau protokolliert und wissenschaftlich durch die Technische Fachhochschule Wildau begleitet. Den Gaserzeuger lieferte die Firma Fluidyne Ltd. (Auckland, NZ). Fluidyne ist auf dem Gebiet der Gaserzeugerentwicklung weltweit fuihrend und besitzt seit 20 Jahren Erfahrungen mit der Produktion von Gaserzeugern.

Mit dem einjährigen Probebetrieb einer $30 \mathrm{~kW}_{\mathrm{el}}$ Anlage bestehend aus einem Fluidyne Gaserzeuger und einem MAN-BHKW ist die grundsätzliche Einsatzfähigkeit unter den herrschenden ökologischen und ökonomischen Bedingungen nachgewiesen. Die wesentlichen Kenngrößen (Massenstrom und Energiebilanz, Wirkungsgrad und Emissionen) der Anlage sind bekannt.

Bei den Fragen und Detaillösungen wurde grundsätzlich der Kontakt zu Firmen und Instituten gesucht, die über spezielle Kenntnisse verfügen. Damit wird die Entwicklung von Komponeneten vermieden, die bereits auf dem Markt verfügbar sind. Durch die Kontakte wurden kommerzielle und wissenschaftliche Partner gefunden, die mit ihrem Wissen und ihren Produkten für die Weiterentwicklung der Gaserzeugertechnik genutzt werden können.

\section{Voraussetzung für den kommerziellen Einsatz von Holzgaserzeugern}

Durch die wirtschaftlichen und umweltrelevanten Veränderungen in der Bundesrepublik ist die Nutzung von Holzgaserzeugern für die Energieproduktion eine Alternative zur konventionellen Energieerzeugung geworden. Die Holzgaserzeuger-Anlagen müssen für den erfolgreichen Einsatz folgende Voraussetzungen erfuillen:

- Leistungsklasse: 300-500 kW Stromproduktion entsprechend einer Feuerungsleistung von 1,5-2,3 MW

- Automatische Prozeßüberwachung und kontinuierlicher Betrieb

- Wartungs- und Bedienungsaufwand max. 1 Std./Tag

- Entsorgungskonzept für die Rückstände: Kon- densat, Ruß und Asche

- Einhaltung der Emissionsgrenzwerte für die Abgase

- Spezifischer Anlagenpreis von max. $4500 \mathrm{DM} / \mathrm{kW}_{\mathrm{el}}$

\section{Betriebserfahrungen}

Am TSZ ist seit Juni 1995 ein Holzvergaser in Betrieb, Die Anlage, bestehend aus einem Gleichstromvergaser und BHKW, liefert pro Stunde 28kW Strom und $48 \mathrm{~kW}$ Wärme. Der Strom wird ins Netz eingespeist und mit der Wärme werden Schulungsräume und Büros beheizt. Die Anlage wird in der Tagesschicht gefahren und hat inzwischen mehr als 800 Betriebsstunden erreicht. Begleitet wird der Dauertest durch Untersuchungen der Umweltverträglichkeit und Wirtschaftlichkeit.

\subsection{Technische Daten der Maschinen}

Holzgaserzeuger 'Pacific Class' Hersteller Fluidyne, Auckland NZ

Typ Festbettvergaser, absteigend

Brennstoff Holz, stückig

Feuchte mac. $30 \%$

Verbrauch $\quad 30-45 \mathrm{~kg} / \mathrm{h}$

Gasproduktion $\quad 25-100 \mathrm{Nm}^{3} / \mathrm{h}$

Energieproduktion $35 \mathrm{~kW}_{\mathrm{el}}$ im Saugbetrieb

Filter

Gravitationabscheidung, Zyklon, Festbettfilter mit Spänen

\section{BHKW MAN NAR $50 \mathrm{M}$}

Leistung

Verfahren

Hubraum/Zylinder

Kompression

$42 \mathrm{~kW}_{\mathrm{el}}, 73 \mathrm{~kW}_{\mathrm{th}}$ (mit Erdgas im Magermix)

Nenndrehzahl Gas-Otto-Motor/4 Takt

5,7 Liter, 6 Zyl. Reihe

$1: 12,5$

$1500 \mathrm{Rpm}$

Die folgende Skizze verdeutlicht das Anlagensystem. Das Holzgas entsteht in dem Reaktor. Oberhalb der Vergasungszone befindet sich der Brennstoffbunker, in dem das Holz getrocknet und pyrolysiert wird. Nach der Vergasung wird das Gas von Partikeln gereinigt und gekühlt. Dafür werden Gravitationfilter, Zyklone und Festbettfilter eingesetzt. Gekühlt wird mit Röhren-

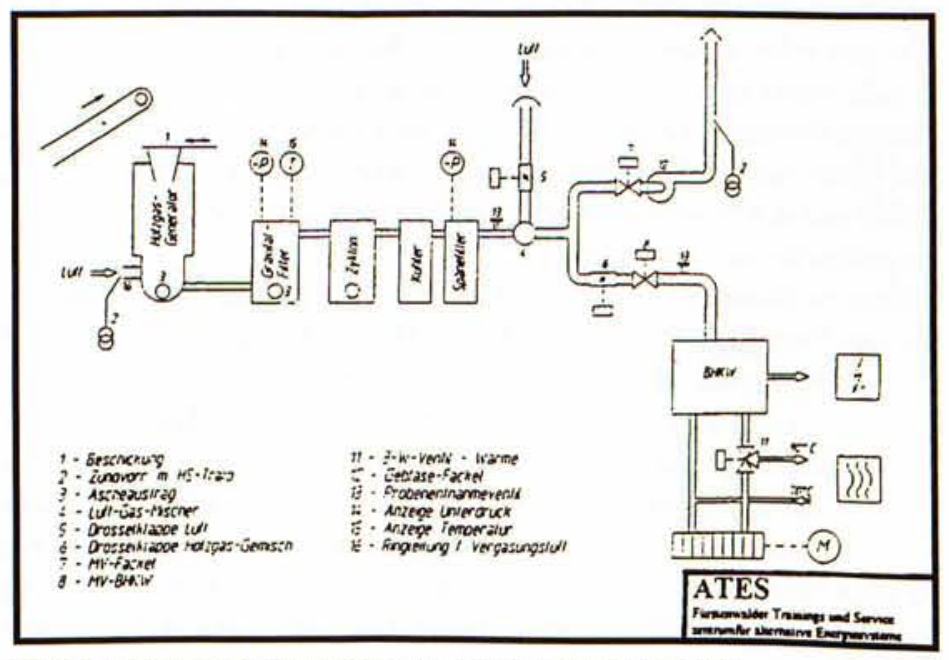

Bild 5.1: Schema der Fluidyne Gaserzeugeranlage am TSZ Fürstenwalde 
kühlern. Dem Gas wird am Mischer die für die Verbrennung im Motor erforderliche Luft beigemischt und dann angesaugt. Parallel zur Gasleitung des Motors ist das Startgebläse installiert. Die Startzeit beträgt 5 Minuten. In der folgenden Tabelle werden die wichtigsten Kenndaten aus dem Dauertest des Holzgaserzeuger-Blockheizkraftwerk-Systems vorgestellt.

\begin{tabular}{|c|c|c|c|}
\hline Parameter & Einheit & \multicolumn{2}{|c|}{ Wert } \\
\hline \multicolumn{4}{|l|}{ Energie } \\
\hline \multicolumn{4}{|c|}{ Spezifische Daten (Mittelwerte) } \\
\hline BHKW Leistung el. & $/ \mathrm{kW}$ & \multicolumn{2}{|l|}{28} \\
\hline BHKW Leistung therm & $/ \mathrm{kW}$ & 48 & \\
\hline \multicolumn{4}{|l|}{ Gaserzeuger } \\
\hline Feuerungsleistung & /kW & \multicolumn{2}{|c|}{125} \\
\hline Leistung therm. & $/ \mathrm{kW}$ & \multicolumn{2}{|l|}{38} \\
\hline Brennstoff & \multicolumn{3}{|c|}{ Holz (unbehandelt) } \\
\hline Brennstoffiverbrauch & $/ \mathrm{kg} / \mathrm{h}$ & \multicolumn{2}{|c|}{31 (wf) } \\
\hline Brennstoffverbrauch & $/ \mathrm{kg} / \mathrm{kWh}$ & \multicolumn{2}{|c|}{$1,1(w f)$} \\
\hline Stromkennzahl & & \multicolumn{2}{|c|}{0,59} \\
\hline \multicolumn{2}{|c|}{ Wirkungsgrad elektrisch } & - & 0,22 \\
\hline \multicolumn{2}{|c|}{ Wirkungsgrad thermisch } & - & 0,38 \\
\hline \multicolumn{2}{|c|}{ Wirkungsgrad gesamt - } & \multicolumn{2}{|c|}{0,60} \\
\hline
\end{tabular}

(Wirkungsgrade bezogen auf den Brennstoffeinsatz bzw. Gesamtanlage)

Brennstoffeuchte

Heizwert Hu (wf)

$\begin{array}{ll}/ \% & 10 \\ / \mathrm{kJ} / \mathrm{kg} & 17500\end{array}$

Austrag bezogen auf den Brennstoff (wf)

Asche Summe

$1,8 \%$

Kondensat

$5,4 \%$

(neutral durch Aktivkohle)

\section{BHKW Abgasmessung}

$\begin{array}{llll}\text { Gaskomponente } & & \text { Wert } & \text { TALuft } \\ \text { CO2 } & / \% & 17,95 & \\ \text { CO } & / \mathrm{mg} / \mathrm{m}^{3} & 2450^{*} & 650 \\ \text { Nox } & / \mathrm{mg} / \mathrm{m}^{3} & 106 & 500 \\ \text { SO2 } & / \mathrm{mg} / \mathrm{m}^{3} & 0 & 400\end{array}$

'Senkung unterhalb TA-Luft techn. möglich

\section{Kondensatanalysen}

Stoff

Absetzbare Stoffe

$\mathrm{pH}$-Wert

CSB

CSB (gereinigt*)

Cyanide

Phenole

$\begin{array}{lll}\text { Einheit } & \text { Wert } & \text { Limit } \\ \text { mg/l } & 0,3 & 6,0 \\ & 8,8 & 9,5 \\ \text { mg/l } & 1900 & \\ \text { mg/l } & 100 & 600 \\ \text { mg/l } & 0.013 & 0,2 \\ \text { mg/1 } & 108 & \\ \text { mg/l } & 63 & 75\end{array}$

Phenole (gereinigt ${ }^{*}$ )

"mit Aktivkohle

Analyse von Holzgas mit Kapillargaschromatograph nach DIN 51872

Labor: GASAG Berliner Gaswerke AG, Probenahme Nov.'95

Kenndaten bei der Probenahme:

Holzgasproduktion: $72 \mathrm{~m}^{3} / \mathrm{h}$

Vollast $=100 \mathrm{~m}^{3} / \mathrm{h}$

Leistung elektrisch: $20 \mathrm{~kW}$ (Netzeinspeisung)

Komponente
Stickstoff $\quad 49$

Kohlenmonoxid $\quad 17$

Wasserstoff $\quad 15$

Kohlendioxid $\quad 12$

Methan $\quad 1,7$

Ethen $\quad 0,3$

Summe der höheren Kohlenwasserstoffe $<0,1$

$\begin{array}{ll}\text { (Rest } \mathrm{H} 2 \mathrm{O} & \text { ca. } 5)\end{array}$

Heizwert $(\mathrm{Hu}) / \mathrm{kJ} / \mathrm{m}^{3} \quad 4530$

Tabelle 5.1: Kenndaten des Fluidyme Gaserzeugeranlage am TSZ Fürstenwalde

\section{Energiebilanz}

Aus der Energiemenge des eingesetzten Brennstoffs kann mit einem Gaserzeuger und BHKW mehr als $20 \%$ Strom erzeugt werden. In dem Energieflußdiagramm sind die möglichen Potentiale aufgefuihrt. Ob eine vollständige Ausnutzung sinnvoll ist, muß der Planer im Einzelfall entscheiden. Bei entsprechendem Wärmebedarf, z. B. Trocknung in Tischlereien, ist die BHKWTechnik und die Nutzung der Gaserzeugerabwärme zu empfehlen.

In einem anderen Fall ist evtl. die reine Stromerzeugung sinnvoll. Dabei sind die Investitionen wesentlich geringer. Auch ist die reine Stromerzeugung bei sonst ungenutzten Brennstoffen trotz des geringen Gesamtwirkungsgrades ökologisch vertretbar.

Die Wirkungsgrade des Holzgas-BHKW's erscheinen in diesem Diagramm kleiner als die bekannten Werte für

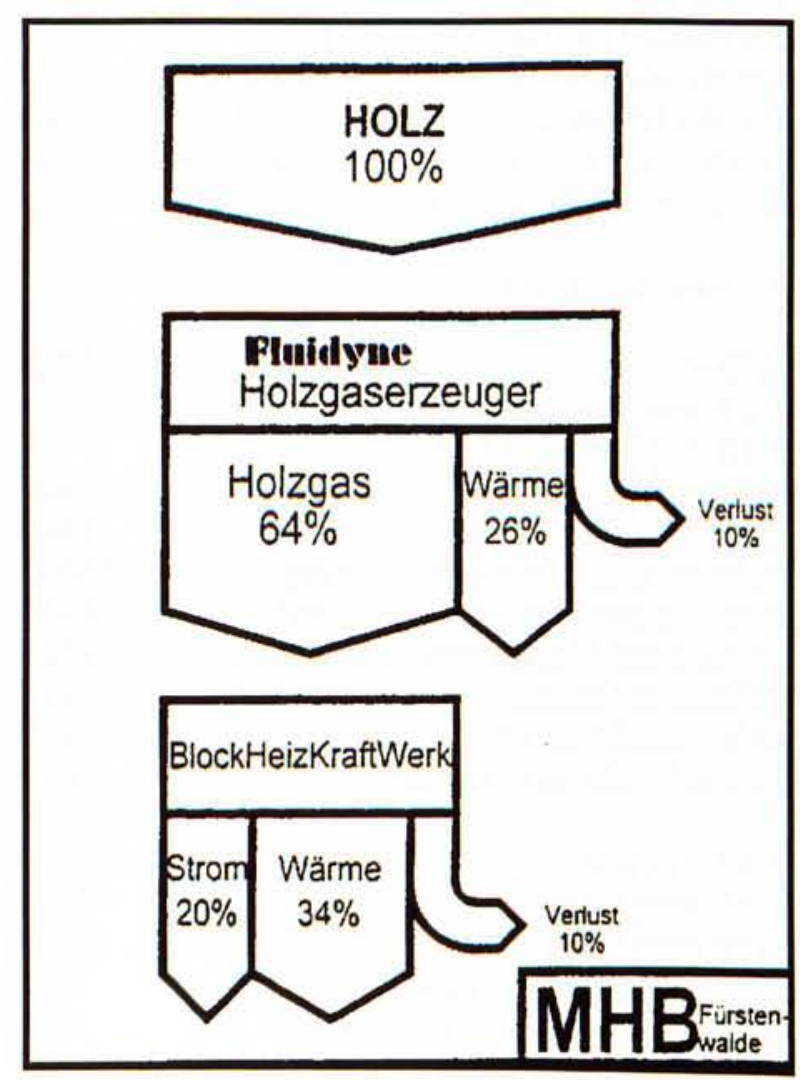

Bild 6.1: Energiebilanz eines Holzgaserzeuger-BHKW System 
Erdgas-BHKKW's. Tatsächlich sind die Wirkungsgrade von Holzgas- und Erdgas- BHKW's aber fast gleich. Im direkten Vergleich muß, wie bei Erdgas, der Heizwert und die Menge an Holzgas als Basis der Berechnung gewählt werden. Für den mechanischen Wirkungsgrad bedeutet dies: $22 \% / 0,64=34 \%$ Wirkungsgrad für das BHKW. Tatsächlich wird z. Zt. von der Demonstrationsanlage ein Wirkungsgrad von $22 \%$ entsprechend $34 \%$ erreicht.

\section{Wirtschaftlichkeit}

Generelle Einschätzungen sind schwierig. Die Anlagen werden individuell konzipiert. In der Regel kann aber bei entsprechendem Brennstoffaufkommen eine wirtschaftlich mögliche Konzeption gefunden werden. Für die Einschätzung sind folgende Punkte wichtig:

- Investitionen für die Gesamtanlage

- Einsparung der Strombezïge von dem EVU

- Brennstoffkosten besonders interessant bei belasteten Hölzern, d. h. bei negativen Brennstoffpreisen

- Wärmenutzung

- Grad der Automatisation. In der Regel ist bei kleinen Anlagen $\left(<100 \mathrm{~kW}_{\mathrm{el}}\right)$ Handbetrieb oder eine Halbautomatik sinnvoll. Nur größere Maschinen können durch ihren Ertrag die Mehrkosten für eine Automatisation erwirtschaften.

Gerade die Wirtschaftlichkeitsbetrachtung hat gezeigt, daß es sinnvoll ist, größere Einheiten von Gaserzeugern zu bauen. Beim up-scaling der Vergaser treten in der Regel Probleme auf. So ist es wichtig, trotz der notwendigen größeren Rostfläche das horizontale Temperaturprofil der Zonen zu erhalten. Dies ist mit der Entwicklung des Mega-Class-Gasifiers auf der Basis des bewährten Pacific-Class-Gasifiers gelungen.

Um die Wirtschaftlichkeit zu verdeutlichen, wurde für den Fluidyne-Mega-Class-Gasifier eine Kalkulation nach einem möglichen Einsatzszenario berechnet und in der folgenden Tabelle aufgeführt:

\subsection{Kostenspezifikation}

Position Einheit

Wert

\subsubsection{Technische Daten}

7.1.1.1 Holzgaserzeuger

$\begin{array}{llr}\text { Feuerungsleistung } & \mathrm{KW} & 2300 \\ \text { Gasproduktion } & \mathrm{m}^{3} / \mathrm{h} & 1380 \\ \text { Heizwert Holz (Wasserfrei) } & \mathrm{kJ} / \mathrm{kg} & 15000 \\ \text { Heizwert Generatorgas } & \mathrm{kJ} / \mathrm{m}^{3} & 4500 \\ \text { Chem. Leistungspotential } & \mathrm{kW} & 1725 \\ \text { Brennstoffverbrauch } & \mathrm{kg} / \mathrm{h}(\mathrm{wf}) & 552 \\ \text { Holzgaserzeuger Wärme } & \mathrm{kW} & 345 \\ \text { Wirkungsgrad Gaserzeuger } & & 0,64\end{array}$

\subsubsection{BHKW}

Wirkungsgrad el.

Wirkungsgrad th.

Leistung el. (Generatorgas) kW

Leistung th. (Generatorgas) kW

7.1.2 EinsatzSzenario

Einsatztage/Jahr
Verfügbarkeit

Betriebsstunden

Betriebsstunden

Brennstoffverbrauch

Brennstoffverbrauch

(bei $20 \%$ Wassergehalt)

Prod. Energiemenge el.

Prod. Energiemenge th.

Strompreis

Wärmepreis

Stromertrag

Wärmeertrag

Ertragssumme

\subsubsection{Investition}

Gaserzeuger

BHKW

Gesamtkosten

spez. Invest. Kosten

\subsubsection{Betriebskosten}

spez. Personal Kosten

Arbeitszeit

Personal

spez. Servicekosten

Service

Kondensatabnahmegebühr

Kondensatmenge

Kondensatentsorgung

Aschabnahmegbiihr

Aschemenge

Ascheentsorgung

Brennstoffannahme

Brennstoffaufbereitung

Brennstoff

Betriebskosten gesamt

verfuigbare Kapitalkosten

Annuität

KreditZinssatz $(8 \%)$

Tilgung

(Amortisation)

DM

DM

DM

$\mathrm{DM} / \mathrm{kW}_{\mathrm{el}}$.

$\mathrm{h} / \operatorname{tag}$

24

$\mathrm{h} / \mathrm{a}$

6426

$\mathrm{kg} / \mathrm{h}$

662

t/a

4256

$\begin{array}{lr}\text { MWh } & 3251 \\ \text { MWh } & 5616 \\ \text { DM/MWh } & 168 \\ \text { DM/MWh } & 65\end{array}$

DM

DM

546261

365061

911322

DM

770000

1245000

2015000

3980

$\begin{array}{lr}\mathrm{DM} / \mathrm{h} & 50 \\ \mathrm{~h} / \mathrm{Tag} & 1,5 \\ \mathrm{DM} & 23625 \\ \mathrm{DM} / \mathrm{MWh}_{\text {el. }} & 50 \\ \mathrm{DM} & 162577 \\ \mathrm{DM} / \mathrm{m}^{3} & 25 \\ \% \mathrm{Br} . \mathrm{st} . & 15 \\ \mathrm{DM} & 15962 \\ \mathrm{DM} / \mathrm{t} & 1000 \\ \% \mathrm{Br} . \mathrm{st} . \text { (wf) } & 2 \\ \mathrm{DM} & 85131 \\ \mathrm{DM} / \mathrm{t} & -50 \\ \mathrm{DM} / \mathrm{t} & 50 \\ \mathrm{DM} & 0,00 \\ \mathrm{DM} & 287296 \\ \mathrm{DM} & 624025 \\ \% & 31 \\ \% & 8 \\ \% & 23 \\ \% & (4,3) \\ \text { Jahre } & \end{array}$

\section{Verfasser}

Dipl.-Phys. Wilfried Richter

MHB GmbH, Fürstenwalde

Prof. Dr.-Ing. Udo Hellwig

Technische Fachhochschule Wildau

Fachbereich Ingenieurwesen/Wirtschaftsingenieurwesen Tel. 03375/507-170 